\title{
S. Louboutin
}

Nagoya Math. J.

Vol. 161 (2001), 171-191

\section{COMPUTATION OF $L(0, \chi)$ AND OF RELATIVE CLASS NUMBERS OF CM-FIELDS}

\author{
STÉPHANE LOUBOUTIN
}

\begin{abstract}
Let $\chi$ be a nontrivial Hecke character on a (strict) ray class group of a totally real number field $\mathbf{L}$ of discriminant $d_{\mathbf{L}}$. Then, $L(0, \chi)$ is an algebraic number of some cyclotomic number field. We develop an efficient technique for computing the exact values at $s=0$ of such abelian Hecke $L$-functions over totally real number fields $\mathbf{L}$. Let $f_{\chi}$ denote the norm of the finite part of the conductor of $\chi$. Then, roughly speaking, we can compute $L(0, \chi)$ in $O\left(\left(d_{\mathbf{L}} f_{\chi}\right)^{0.5+\epsilon}\right)$ elementary operations. We then explain how the computation of relative class numbers of CM-fields boils down to the computation of exact values at $s=0$ of such abelian Hecke $L$-functions over totally real number fields L. Finally, we give examples of relative class number computations for CM-fields of large degrees based on computations of $L(0, \chi)$ over totally real number fields of degree 2 and 6.
\end{abstract}

\section{$\S 1$. Introduction}

Let $\chi$ be a nontrivial Hecke character on a (strict) ray class group of a totally real number field $\mathbf{L}$. Then, $L(0, \chi)$ is an algebraic number of some cyclotomic number field $\mathbf{Q}(\chi)$. The first aim of this paper is to develop a practical and efficient technique for computing the exact values at $s=0$ of such abelian Hecke $L$-functions over totally real number fields $\mathbf{L}$, i.e. for computing the exact values of the rational coordinates of this algebraic number $L(0, \chi)$ in a given basis of this cyclotomic field $\mathbf{Q}(\chi)$ (see Theorem 7 and Remark 8). We apply this technique to the efficient computation of relative class numbers of CM-fields (see Theorem 9). We finally give in Section 5 examples of computation of relative class numbers of CM-fields of large degrees based on computations of the exact values of values at $s=0$ of $L$-functions over totally real number fields of degree 2 and 6 . We also refer the reader to [CK], [Lef], [LP] and [Park] for other examples of the use of the present technique. Let us point out that our method applies equally well to the computation of $L(-n, \chi), n \geq 0$. We restrict ourselves to the

Received May 10, 1999.

1991 Mathematics Subject Classification: Primary 11R29, 11R21, 11 Y35. 
computation of $L(0, \chi)$ because we only aim at applying our method for computing relative class numbers of CM-fields.

To compute such exact values we fix a $\mathbf{Z}$-basis $\mathcal{B}$ of the ring of algebraic integers $\mathbf{Z}[\chi]$ of the cyclotomic field $\mathbf{Q}(\chi)$ generated by the values of $\chi$ and we compute the coordinates of $L(0, \chi)$ in this basis $\mathcal{B}$. Since these coordinates are rational numbers whose denominators are bounded beforehand (see Theorem 2), to compute their exact values we only have to compute sufficiently good numerical approximations of them. By expressing these coordinates as linear combinations of finitely many values $L\left(0, \chi^{l}\right)$ for some $l \geq 1$ (see (6) and (7)), we will reduce the computation of approximations of these coordinates to the computation of sufficiently good approximations of values of several $L\left(0, \chi^{l}\right)$. We will finally remind the reader of the technique developed in [Lou6] for computing as good as desired numerical approximations of values at $s=0$ of such abelian Hecke $L$-functions.

Notation. Throughout this paper, we let $\mathbf{L}$ be a totally real number field of degree $m \geq 1$ and $\mathcal{F}$ be an integral ideal of $\mathbf{L}$. We write $\mathbf{L}_{\mathcal{F}}$ for the set of all totally positive elements $\alpha$ of $\mathbf{L}$ such that $\nu_{\mathcal{P}}(\alpha-1) \geq \nu_{\mathcal{P}}(\mathcal{F})$ for all primes $\mathcal{P}$ of $\mathbf{L}$ which divide $\mathcal{F}$. The (strict) ray class group $\bmod \mathcal{F}$, which we denote by $\mathbf{R}_{\mathcal{F}}(\mathbf{L})$, is defined to be the quotient of the group of fractional ideals of $\mathbf{L}$ generated by the primes not dividing $\mathcal{F}$, by the the subgroup consisting of all principal ideals $(\alpha)$ with $\alpha \in \mathbf{L}_{\mathcal{F}}$.

We let $\chi$ denote a primitive character or order $n_{\chi}>1$ on $\mathbf{R}_{\mathcal{F}}(\mathbf{L})$ and set $f_{\chi}=N_{\mathbf{L} / \mathbf{Q}}(\mathcal{F})$, the norm of the finite part of the conductor of $\chi$. We let $\mathbf{M}_{\chi} / \mathbf{L}$ denote the cyclic extension of degree $n_{\chi}$ and conductor $\mathcal{F}$ associated with $\chi$ and we let $w_{\chi}$ denote the number of roots of unity of $\mathbf{M}_{\chi}$. Notice that $\phi\left(w_{\chi}\right)$ divides the degree $m n_{\chi}$ of $\mathbf{M}_{\chi}$. Thus, $w_{\chi} \leq \phi^{2}\left(w_{\chi}\right) \leq m^{2} n_{\chi}^{2}$. We set $\zeta_{\chi}=\exp \left(2 \pi i / n_{\chi}\right), \mathbf{Q}(\chi)=\mathbf{Q}\left(\zeta_{\chi}\right)$. We let $\phi_{\chi}=\phi\left(n_{\chi}\right)$ and $\mathbf{Z}[\chi]=\mathbf{Z}\left[\zeta_{\chi}\right]$ denote the degree and the ring of algebraic integers of the cyclotomic field $\mathbf{Q}(\chi)$, respectively. Finally, for any $l$ relatively prime to $n_{\chi}$ we let $\sigma_{l}$ denote the $\mathbf{Q}$-automorphism of $\mathbf{Q}(\chi)$ which is defined by $\sigma_{l}\left(\zeta_{\chi}\right)=\zeta_{\chi}^{l}$.

\section{$\S 2$. Prerequisite on $L(0, \chi)$}

Let $\chi$ be a primitive Hecke character on a ray class group $\mathbf{R}_{\mathcal{F}}(\mathbf{L})$ modulo $\mathcal{F}$, an integral ideal of number field $\mathbf{L}$ of degree $m$. To begin with, we notice that if $L(0, \chi) \neq 0$ then $\mathbf{L}$ is totally real and $\chi$ is ramified at the $m$ infinite places of $\mathbf{L}$. From now on, we assume that $\chi$ is ramified at all the $m$ infinite places of a totally real number field $\mathbf{L}$ of degree $m$. 
For each $\mathcal{I} \in \mathbf{R}_{\mathcal{F}}(\mathbf{L})$, the partial zeta function of $\mathcal{I}$ is defined by

$$
\zeta_{\mathcal{F}}(\mathcal{I}, s)=\sum_{\mathcal{A}}\left(N_{\mathbf{L} / \mathbf{Q}}(\mathcal{A})\right)^{-s} \quad(\Re(s)>1)
$$

where the summation is taken over all integral ideals $\mathcal{A}$ of $\mathbf{L}$, prime to $\mathcal{F}$, which belong to the class of $\mathcal{I}$, and where $N_{\mathbf{L} / \mathbf{Q}}(\mathcal{A})$ denotes the norm from $\mathbf{L}$ to $\mathbf{Q}$ of $\mathcal{A}$. Notice that $\zeta_{\mathcal{F}}(\mathcal{I}, s)$ depends only on the ray class of $\mathcal{I}$ modulo $\mathcal{F}$. If $\mathbf{L}=\mathbf{Q}, \mathcal{F}=(f)$ and $\mathcal{I}=(a), a \geq 1$ then

$$
\zeta_{\mathcal{F}}(\mathcal{I}, s)=\zeta_{(f)}((a), s)=f^{-s} \zeta(a / f, s)
$$

where

$$
\zeta(b, s)=\sum_{k \geq 0}(b+k)^{-s} \quad(0<b \leq 1 \text { and } \Re(s)>1)
$$

denotes Hurwitz's zeta function. We have $\zeta(b, 0)=\frac{1}{2}-b$, so that $\zeta_{(f)}((a), 0)$ is a rational number. This result was generalized by Siegel and Klingen who proved that $\zeta_{\mathcal{F}}(\mathcal{I}, 0)$ is rational. We notice that explicit denominators for values at $s=0$ of partial zeta functions seem to be known only in the cases where $\mathbf{L}$ is either the rational number field or a real quadratic number field:

Proposition 1. (See [CS1, Th.8].) Assume that $\mathbf{L}$ is a real quadratic field. Then, $12 N_{\mathbf{L} / \mathbf{Q}}(\mathcal{F}) \zeta_{\mathcal{F}}(\mathcal{I}, 0)$ is a rational integer. Moreover, if $\mathcal{F}=(f)$ for some positive rational integer $f \geq 1$, then $12 f \zeta_{\mathcal{F}}(\mathcal{I}, 0)$ is a rational integer.

Let now $\chi$ be a primitive character of order $n_{\chi}>1$ on the (strict) ray class group $\mathbf{R}_{\mathcal{F}}(\mathbf{L})$ modulo $\mathcal{F}$ and let

$$
L(s, \chi)=\sum_{\mathcal{I}} \chi(\mathcal{I}) \zeta_{\mathcal{F}}(\mathcal{I}, s)
$$

(where $\mathcal{I}$ ranges over a set of representatives of the ray class group modulo $\mathcal{F}$ ) be the abelian Hecke $L$-series associated to $\chi$. Setting

$$
a_{n}(\chi)=\sum_{N_{\mathbf{L} / \mathbf{Q}}(\mathcal{A})=n} \chi(\mathcal{A})
$$

(this sum ranges over all the non zero integral ideals of $\mathbf{L}$ of norm $n$ ) we have

$$
L(s, \chi)=\sum_{n \geq 1} \frac{a_{n}(\chi)}{n^{s}} \quad(\Re(s)>1)
$$


According to (1) and to Siegel-Klingen's Theorem, $L(0, \chi)$ is in $\mathbf{Q}(\chi)$ and for any rational integer $l$ relatively prime to $n_{\chi}$ we have

$$
\sigma_{l}(L(0, \chi))=L\left(0, \chi^{l}\right)
$$

If $\mathbf{L}=\mathbf{Q}$, then $\chi$ is a Dirichlet character modulo $f>1$ of order $n_{\chi}>1$ and we have $L(s, \chi)=f^{-s} \sum_{a=1}^{f-1} \chi(a) \zeta(a / f, s)$. Hence, setting $\zeta(a, c, s)=$ $c^{s-1} \zeta(\{a c / f\}, s)-\zeta(\{a / f\}, s)$ for $a \geq 1, c \geq 1$ and $\operatorname{gcd}(a c, f)=1$, we have $\sum_{a=1}^{f-1} \chi(a) \zeta(a, c, s)=\left(\bar{\chi}(c) c^{s-1}-1\right) f^{s} L(s, \chi)$, which yields

$$
(c-\bar{\chi}(c)) L(0, \chi)=-c \sum_{a=1}^{f-1} \chi(a) \zeta(a, c, 0)=-\sum_{a=1}^{f-1}[a c / f] \chi(a) \in \mathbf{Z}[\chi]
$$

(where $[x] \in \mathbf{Z}$ denotes the greatest rational integer less than or equal to a real $x$ and where $\{x\}=x-[x] \in[0,1$ [ denotes the fractional part of $x)$. Hence, $\operatorname{gcd}(c, f)=1$ and $\chi(c)=1$ imply $(c-1) L(0, \chi) \in \mathbf{Z}[\chi]$. In particular, if $d=\operatorname{gcd}\{c-1, c \in \operatorname{ker} \chi\}$ then $d L(0, \chi) \in \mathbf{Z}[\chi]$. However, in this situation $\mathbf{M}_{\chi}$ is the subfield of $\mathbf{Q}\left(\zeta_{f}\right)$ fixed by ker $\chi$ (i.e. $\alpha \in \mathbf{Q}\left(\zeta_{f}\right)$ is in $\mathbf{M}_{\chi}$ if and only if $\sigma_{c}(\alpha)=\alpha$ for all $c \in \operatorname{ker} \chi\left(\right.$ where $\left.\sigma_{c}\left(\zeta_{f}\right)=\zeta_{f}^{c}\right)$ ). Now, let $\zeta_{d}$ be any $d$ th primitive complex root of unity. Then $d$ divides $f$ (for $1+f \in \operatorname{ker} \chi$ ), hence $\zeta_{d} \in \mathbf{Q}\left(\zeta_{f}\right)$ and $\sigma_{c}\left(\zeta_{d}\right)=\zeta_{d}^{c}=\zeta_{d}$ for all $c$ 's in ker $\chi$. Hence, $\zeta_{d}$ which is fixed by ker $\chi$ is in $\mathbf{M}_{\chi}$. Therefore, $d$ divides $w_{\chi}$ and $w_{\chi} L(0, \chi)$ is in $\mathbf{Z}[\chi]$. Hence, we have proved that $w_{\chi} L(0, \chi) \in \mathbf{Z}[\chi]$ for any Dirichlet character $\chi$. This result was generalized to arbitrary characters $\chi$ on ray class groups of totally real number fields $\mathbf{L}$ :

Theorem 2. (See $[\mathrm{CS} 2]$ and $[\mathrm{Cas}]$.) It holds $w_{\chi} L(0, \chi) \in \mathbf{Z}[\chi]$.

Let $\mathcal{B}=\left\{\epsilon_{1}, \cdots, \epsilon_{\phi_{\chi}}\right\}$ be any $\mathbf{Z}$-basis of $\mathbf{Z}[\chi]$. Let $\mathcal{B}^{\perp}=\left\{\theta_{1}, \cdots, \theta_{\phi_{\chi}}\right\}$ be its dual basis relative to the trace form (see [Lan, Prop. 2 page 58]), hence

$$
\operatorname{Tr}_{\mathbf{Q}(\chi) / \mathbf{Q}}\left(\epsilon_{k} \theta_{l}\right)=\delta_{k, l}= \begin{cases}1 & \text { if } k=l \\ 0 & \text { if } k \neq l\end{cases}
$$

and set

$$
M\left(\mathcal{B}^{\perp}\right)=\max _{\substack{1 \leq l \leq n_{\chi}, \operatorname{gcd}\left(l, n_{\chi}\right)=1 \\ 1 \leq j \leq \phi \chi}}\left|\sigma_{l}\left(\theta_{j}\right)\right| .
$$

Notice that if $n_{\chi}=\prod_{i=1}^{r} p_{i}^{e_{i}}$ and if

$$
\mathcal{B}_{i}^{\perp}=\left\{\theta_{l}(i) ; 1 \leq l \leq \phi\left(p_{i}^{e_{i}}\right)\right\}
$$


is the dual basis relative to the trace form $\operatorname{Tr}_{\mathbf{Q}\left(\zeta_{p_{i}}\right) / \mathbf{Q}}$ of a $\mathbf{Z}$-basis

$$
\mathcal{B}_{i}=\left\{\epsilon_{k}(i) ; 1 \leq k \leq \phi\left(p_{i}^{e_{i}}\right)\right\}
$$

of the ring of algebraic integers $\mathbf{Z}\left[\zeta_{p_{i}} e_{i}\right]$ of $\mathbf{Q}\left(\zeta_{p_{i}} e_{i}\right)$, then

$$
\mathcal{B}=\left\{\epsilon_{\vec{k}}=\prod_{i=1}^{r} \epsilon_{k_{i}}(i) ; 1 \leq k_{i} \leq \phi\left(p_{i}^{e_{i}}\right)\right\}
$$

is a $\mathbf{Z}$-basis of the ring of algebraic integers $\mathbf{Z}\left[\zeta_{f}\right]$ of $\mathbf{Q}\left(\zeta_{f}\right)$ and

$$
\mathcal{B}^{\perp}=\left\{\theta_{\vec{l}}=\prod_{i=1}^{r} \theta_{l_{i}}(i) ; 1 \leq l_{i} \leq \phi\left(p_{i}^{e_{i}}\right)\right\}
$$

is its dual basis relative to the trace form $\operatorname{Tr}_{\mathbf{Q}\left(\zeta_{f}\right) / \mathbf{Q}}$ which satisfies

$$
M\left(\mathcal{B}^{\perp}\right) \leq \prod_{i=1}^{r} M\left(\mathcal{B}_{i}^{\perp}\right)
$$

Therefore, we only have to explain the construction of dual bases in the case of cyclotomic fields of prime power conductors. Now, noticing that

$$
\operatorname{Tr}_{\mathbf{Q}\left(\zeta_{p^{s}}\right) / \mathbf{Q}}\left(\zeta_{p^{s}}^{l}\right)= \begin{cases}0 & \text { if } p^{s-1} \text { does not divide } l \\ -p^{s-1} & \text { if } p^{s-1} \text { divides } l \text { but } p^{s} \text { does not divide } l \\ p^{s}-p^{s-1} & \text { if } p^{s} \text { divides } l\end{cases}
$$

we have:

LEMMA 3. Let $\phi$ denote Euler's function. Let $p$ be a prime. The dual basis of the $\mathbf{Z}$-basis $\mathcal{B}=\left\{\zeta_{p^{s}}^{k} ; 1 \leq k \leq \phi\left(p^{s}\right)\right\}$ of the ring of algebraic integers of $\mathbf{Q}\left(\zeta_{p^{s}}\right)$ is $\mathcal{B}^{\perp}=\left\{\theta_{l} ; 1 \leq l \leq \phi\left(p^{s}\right)\right\}$ where

$$
\theta_{l}=\frac{1-\zeta_{p}^{1+n(l)}}{p^{s}} \zeta_{p^{s}}^{-l} \text { with } n(l)=\left[\frac{l-1}{p^{s-1}}\right] \in\{0, \cdots, p-2\} .
$$

In particular, $\theta_{l}=2^{1-s} \zeta_{2^{s}}^{-l}$ if $p=2$, and $\theta_{l}=\left(\zeta_{p}^{-l}-1\right) / p$ if $s=1$. Notice that $M\left(\mathcal{B}^{\perp}\right) \leq 2 / p \leq 1$. Therefore, for any $n>2$ there exists some $\mathbf{Z}$-basis of $\mathcal{B}$ of $\mathbf{Z}\left[\zeta_{n}\right]$ such that $M\left(\mathcal{B}^{\perp}\right) \leq 1$. 
THEOREM 4. Let $\mathcal{F}$ be a non zero integral ideal of a number field $\mathbf{L}$ of degree $m \geq 1$. Let $\chi$ be a primitive character on the (strict) ray class group $\mathbf{R}_{\mathcal{F}}(\mathbf{L})$ modulo $\mathcal{F}$ and let $f_{\chi}=N_{\mathbf{L} / \mathbf{Q}}(\mathcal{F})$ denote the norm of the finite part of the conductor of $\chi$. Let $\mathcal{B}=\left\{\epsilon_{1}, \cdots, \epsilon_{\phi_{\chi}}\right\}$ be a $\mathbf{Z}$-basis of $\mathbf{Z}[\chi]$ and let $\mathcal{B}^{\perp}=\left\{\theta_{1}, \cdots, \theta_{\phi_{\chi}}\right\}$ be its dual basis relative to the trace form. Define rational integers $b_{\chi}(k)$ by

$$
w_{\chi} L(0, \chi)=\sum_{k=1}^{\phi_{\chi}} b_{\chi}(k) \epsilon_{k} \in \mathbf{Z}[\chi]
$$

(see Theorem 2). We have

$$
b_{\chi}(k)=w_{\chi} \sum_{\substack{l=1 \\ \operatorname{gcd}\left(l, n_{\chi}\right)=1}}^{n_{\chi}} \sigma_{l}\left(\theta_{k}\right) L\left(0, \chi^{l}\right)
$$

and these coordinates $b_{\chi}(k)$ are rational integers of reasonable size:

$$
\left|b_{\chi}(k)\right| \leq 2 w_{\chi} M\left(\mathcal{B}^{\perp}\right) \sqrt{d_{\mathbf{L}} f_{\chi}}\left(\frac{e}{2 \pi m} \log \left(d_{\mathbf{L}} f_{\chi}\right)\right)^{m} .
$$

Proof. To get (7), use

$$
b_{\chi}(k)=\operatorname{Tr}_{\mathbf{Q}(\chi) / \mathbf{Q}}\left(\theta_{k} w_{\chi} L(0, \chi)\right) .
$$

To get (8), use

$$
|L(0, \chi)| \leq 2 \sqrt{d_{\mathbf{L}} f_{\chi}}\left(\frac{e}{2 \pi m} \log \left(d_{\mathbf{L}} f_{\chi}\right)\right)^{m}
$$

(see [Lou7] and use (10) below).

$\S 3$. Numerical computation of the exact values of the coordinates $b_{\chi}(k)$ of $L(0, \chi)$

3.1. Numerical computation of approximations of $L(0, \chi)$ Set $A_{\chi}=\sqrt{d_{\mathbf{L}} f_{\chi} / \pi^{m}}$. Since

$$
L(0, \chi)=W_{\chi} A_{\chi} \pi^{-m / 2} L(1, \bar{\chi}),
$$

(where $W_{\chi}$ is a complex number of asolute value one, the so called Artin root number associated with $\chi$ ), numerical computation of approximations of $L(0, \chi)$ boils down to numerical computation of approximations of $L(1, \chi)$ and we recall: 
Theorem 5. (See [Lou6].) For $m \geq 1, j \in\{1,2\}, B>0$ and $\alpha>1$ we set

$$
K_{m, j}(B)=\frac{1}{2 \pi i} \int_{\alpha-i \infty}^{\alpha+i \infty} \Gamma^{m}(s) \frac{B^{2-2 s}}{s-(1 / j)} d s
$$

(which is real and does not depend on $\alpha>1$ ). Then

$$
0 \leq K_{m, 2}(B) \leq K_{m, 1}(B) \leq m e^{-B^{2 / m}},
$$

Let $\chi$ be a primitive abelian Hecke character over a totally real field $\mathbf{L}$ of degree $m$. Assume that $\chi$ is ramified at all the $m$ infinite places of $\mathbf{L}$. Then

$$
\begin{aligned}
L(0, \chi)= & \frac{A_{\chi}}{\pi^{m / 2}} \sum_{n \geq 1} \frac{a_{n}(\chi)}{n} K_{m, 2}\left(n / A_{\chi}\right) \\
& +\frac{A_{\chi} W_{\chi}}{\pi^{m / 2}} \sum_{n \geq 1} \frac{\overline{a_{n}(\chi)}}{n} K_{m, 1}\left(n / A_{\chi}\right) .
\end{aligned}
$$

According to (11), these series are absolutely and rapidly convergent.

Finally, let the $a_{n}(\chi)$ 's be as in (2) and set

$$
S_{M}(\chi)=\frac{A_{\chi}}{\pi^{m / 2}} \sum_{n=1}^{M} \frac{a_{n}(\chi)}{n} K_{m, 2}\left(n / A_{\chi}\right)+\frac{A_{\chi} W_{\chi}}{\pi^{m / 2}} \sum_{n=1}^{M} \frac{\overline{a_{n}(\chi)}}{n} K_{m, 1}\left(n / A_{\chi}\right) .
$$

For any positive integer $M \geq\left(m^{2} / 2\right)^{m / 2} A_{\chi}$ we have (see [Lou1, Proof of Proposition 2]):

$$
\left|L(0, \chi)-S_{M}(\chi)\right| \leq \frac{2 m}{\pi^{m / 2}} A_{\chi}\left(\log (M e)+m^{2} / 2\right)^{m} e^{-\left(M / A_{\chi}\right)^{2 / m}} .
$$

It now remains to explain how we compute numerically $K_{m, 1}(B)$ and $K_{m, 2}(B)$ for $B>0$. We give a precise result for the case $m=2$ (and refer the reader to [Lou2] and [Lou6] for $m>2$ ):

Theorem 6. (See [Lou6].)

Let $\gamma=0.577215664901532 \cdots$ denote Euler's constant and set $A_{1}=1, A_{2}=\pi B$. For $B>0$, we have:

$$
K_{2, j}(B)=A_{j}+4 \sum_{n \geq 0}\left(\gamma+\log B-\frac{1}{2 n+3-j}-\sum_{k=1}^{n} \frac{1}{k}\right) \frac{B^{2 n+2}}{(2 n+3-j)(n !)^{2}}
$$


and for any integer $M \geq 0$ we have $\left|R_{j}(M)\right| \leq 2 B^{2 M+3} /(M+1)(M !)^{2}$ where

$$
R_{j}(M) \stackrel{\text { def }}{=} \sum_{n>M}\left(\gamma+\log B-\frac{1}{2 n+3-j}-\sum_{k=1}^{n} \frac{1}{k}\right) \frac{B^{2 n+2}}{(2 n+3-j)(n !)^{2}} .
$$

Of course, one must finally know how to compute the coefficients $a_{n}(\chi)$. Since $n \mapsto a_{n}(\chi)$ is multiplicative, one needs only explain how to compute $a_{p^{k}}(\chi)$ (and we refer the reader to Proposition 11 for such an example).

\subsection{Numerical computation of the exact values of the $b_{\chi}(k)$}

THEOREM 7. Let $\lambda>1, n>1$ and a $\mathbf{Z}$-basis $\mathcal{B}$ of the ring of algebraic integers of the cyclotomic field $\mathbf{Q}\left(\zeta_{n}\right)$ be given. Let $M\left(\mathcal{B}^{\perp}\right)$ be as in $(5)$. Let $\chi$ range over the primitive characters of order $n_{\chi}=n$ on (strict) ray class groups and let the $b_{\chi}(k)$ 's be as in (6). We have

$$
\begin{aligned}
& \left|b_{\chi}(k)-w_{\chi} \sum_{\substack{l=1 \\
\operatorname{gcd}\left(l, n_{\chi}\right)=1}}^{n_{\chi}} \sigma_{l}\left(\theta_{k}\right) S_{M}\left(\chi^{l}\right)\right| \\
& \leq \frac{2 m w_{\chi} M\left(\mathcal{B}^{\perp}\right)}{\pi^{m / 2}} \frac{A_{\chi}\left(\log (M e)+m^{2} / 2\right)^{m}}{e^{\left(M / A_{\chi}\right)^{2 / m}}} \\
& =O\left(\frac{\log ^{m} A_{\chi}}{A_{\chi}^{\lambda-1}}\right) \text { for } M \geq A_{\chi}\left(\lambda \log A_{\chi}\right)^{m / 2} \geq 2 A_{\chi} .
\end{aligned}
$$

Therefore, for $A_{\chi}$ large enough, the coordinates $b_{\chi}(k)$ in the basis $\mathcal{B}$ of the algebraic integer $w_{\chi} L(0, \chi) \in \mathbf{Z}[\chi]$ are rational integers which can be determined in $O\left(A_{\chi}^{0.5+\epsilon}\right)$ elementary operations by computing the $\phi(n)$ approximations $S_{M}\left(\chi^{l}\right)$ for $M$ equal to the least integer greater than or equal to $A_{\chi}\left(\lambda \log A_{\chi}\right)^{m / 2}$ and for $l$ in the range $1 \leq l \leq n$ and $\operatorname{gcd}(l, n)=1$.

Proof. According to (12), $M \geq A_{\chi}\left(\lambda \log A_{\chi}\right)^{m / 2} \geq 2 A_{\chi}$ implies

$$
\left|L\left(0, \chi^{l}\right)-S_{M}\left(\chi^{l}\right)\right| \leq \frac{2 m A_{\chi}}{\pi^{m / 2}} \frac{\left(\log (M e)+m^{2} / 2\right)^{m}}{e^{\left(M / A_{\chi}\right)^{2 / m}}}=O\left(\frac{\log ^{m} A_{\chi}}{A_{\chi}^{\lambda-1}}\right)
$$

and the desired result according to (7). 
Remarks 8. Here we assume that $W_{\chi}$ is known beforehand, for its computation from its definition requires more than $f_{\chi}$ elementary operations. However, for certain classes of characters $W_{\chi}$ is indeed known beforehand (see [FQ], [Fro], [Lou8], (22) and (24) in Section 5). In the case where $W_{\chi}$ it is not known beforehand we explained in [Lou4], [Lou5] and [Lou6, Section 5] how to compute at the same time numerical approximations of $W_{\chi}$ and $L(0, \chi)$ to end up with a practical technique for computing the exact value of $L(0, \chi)$ which conjecturally requires only $A_{\chi}^{0.5+\epsilon}$ elementary operations.

\subsection{A few words on other methods for computing $L(0, \chi)$}

It is known that if $\chi$ is a primitive character on $\mathbf{R}_{\mathcal{F}}(\mathbf{L})$ which is ramified at all the infinite real places of $\mathbf{L}$, then $L(0, \chi)$ is a non-zero complex algebraic number and that Shintani's method provides us with explicit formulae for $L(0, \chi)$ (see [Shi]. See also [Pes]). However, even in the simplest case where $\mathbf{L}$ is a real quadratic number field (and letting $\epsilon_{\mathbf{L}}^{+}=\left(x_{\mathbf{L}}+y_{\mathbf{L}} \sqrt{d_{\mathbf{L}}}\right) / 2>1$ and $h_{\mathbf{L}}^{+}$denote the fundamental totally positive unit and the narrow class number of $\mathbf{L}$ ), Shintani's formulae are of no practical use to compute numerically the exact value of $L(0, \chi)$. Indeed, in the simplest case where $h_{\mathbf{L}}^{+}=1$, Shintani's method yields (see [Hid, Section $2.5])$ :

$$
L(0, \chi)=\sum_{\alpha \in \mathbf{X}_{\mathcal{F}}(\mathbf{L})} r_{\alpha} \chi((\alpha) \mathcal{F})
$$

where the $r_{\alpha}$ are rational numbers and where

$$
\mathbf{X}_{\mathcal{F}}(\mathbf{L})=\left\{\alpha=x_{1}+x_{2} \epsilon_{\mathbf{L}}^{+} \in \mathcal{F}^{-1} ; x_{i} \in \mathbf{Q}, 0<x_{1} \leq 1,0 \leq x_{2}<1\right\}
$$

contains $y_{\mathbf{L}} N_{\mathbf{L} / \mathbf{Q}}(\mathcal{F})$ elements. However, if $h_{\mathbf{L}}^{+}=1$ then according to the Brauer-Siegel theorem $y_{\mathbf{L}}$ is so huge that we cannot compute the finite sum (15) (see Example 1 in Section 5.1) and Shintani's method can only be used for real totally real fields $\mathbf{L}$ with small fundamental units (see [Oka1] and [Oka2] for practical computations).

It is worth pointing out that using continued fractions, Zagier came up with a much more efficient version of Shintani's method for real quadratic fields (see [Zag1], [Zag2] and [Zag3]. See also [LO, Section 5.4] for an example of the use of Zagier's results). However, if a given real quadratic field $\mathbf{L}$ is fixed, then Zagier's method requires the computer to sum up at least $c_{\mathbf{L}} N_{\mathbf{L} / \mathbf{Q}}(\mathcal{F})$ terms in some formula to compute $L(0, \chi)$ (for some suitable constant $c_{\mathbf{L}}$ which depends on $\mathbf{L}$ only), which is useless if $N_{\mathbf{L} / \mathbf{Q}}(\mathcal{F})$ is large 
(see Example 2 in Section 5.1). Notice also that Zagier gave all the details of his method only for characters unramified at all the finite places of $\mathbf{L}$. Moreover, we do not know of any practical generalization of Zagier's improvements for base fields $\mathbf{L}$ of degree $m>3$. The method we developed here enables us to compute efficiently the exact value of $L(0, \chi)$ by computing only few terms in some absolutely and rapidly convergent series (see Theorem 7 to see how dramatic an improvement on Shintani's method ours yields).

\section{$\S 4$. Relative class numbers and $L$-functions at $s=0$}

Throughout this section, $\mathbf{N}$ denotes a CM-field, i.e. $\mathbf{N}$ is a totally imaginary number field which is a quadratic extension of its maximal totally real subfield $\mathbf{N}^{+}$. Let $n$ denote the degree of $\mathbf{N}^{+}$. Let $h_{\mathbf{N}}^{-}, Q_{\mathbf{N}} \in\{1,2\}$ and $w_{\mathbf{N}}$ denote the relative class number of $\mathbf{N}$, the Hasse unit index of $\mathbf{N}$ and the number of roots of unity in $\mathbf{N}$, respectively. Let also $\zeta_{\mathbf{N}}$ and $\zeta_{\mathbf{N}^{+}}$denote the Dedekind zeta functions of $\mathbf{N}$ and $\mathbf{N}^{+}$, respectively. We have (see [Wa]):

$$
h_{\mathbf{N}}^{-}=Q_{\mathbf{N}} w_{\mathbf{N}} 2^{-n}\left(\zeta_{\mathbf{N}} / \zeta_{\mathbf{N}^{+}}\right)(0)
$$

Let $\mathbf{L}$ be any subfield of $\mathbf{N}^{+}$such that the extension $\mathbf{N} / \mathbf{L}$ is abelian, and let $m$ denote the degree of $\mathbf{L}$. We thus have the following lattice of subfields:

$$
\mathbf{Q} \frac{m}{-} \mathbf{L} \frac{n / m}{\mathbf{N}^{+}} \stackrel{2}{-} \mathbf{N}
$$

Notice that we can always choose $\mathbf{L}=\mathbf{N}^{+}$. However, the smaller is the degree $m$ of $\mathbf{L}$ the more efficient is our technique for computing $h_{\mathbf{N}}^{-}$. Therefore, we will choose $\mathbf{L}=\mathbf{Q}$ whenever $\mathbf{N}$ is abelian, whereas we will choose for $\mathbf{L}$ the only real quadratic subfield of $\mathbf{N}$ over which $\mathbf{N}$ is cyclic whenever $\mathbf{N}$ is a dihedral CM-field. We let $X_{\mathbf{N} / \mathbf{L}}$ denote the group of primitive abelian Hecke characters associated with the abelian extension $\mathbf{N} / \mathbf{L}$ and set $X_{\mathbf{N} / \mathbf{L}}^{-}=X_{\mathbf{N} / \mathbf{L}} \backslash X_{\mathbf{N}^{+} / \mathbf{L}}$.

Using (16) and the factorisations of the Dedekind zeta functions $\zeta_{\mathbf{N}}$ and $\zeta_{\mathbf{N}^{+}}$in terms of products of Hecke $L$-functions, we get:

$$
h_{\mathbf{N}}^{-}=Q_{\mathbf{N}} w_{\mathbf{N}} \prod_{\chi \in X_{\mathbf{N} / \mathbf{L}}^{-}} 2^{-m} L(0, \chi)
$$

(notice that there are $n / m$ characters in $X_{\mathbf{N} / \mathbf{L}}^{-}$). Now, let us say that $\chi^{\prime} \in$ $X_{\mathbf{N} / \mathbf{L}}$ is equivalent to $\chi \in X_{\mathbf{N} / \mathbf{L}}$ if there exists $l$ relatively prime to the 
order $n_{\chi}$ of $\chi$ such that $\chi^{\prime}=\chi^{l}$. Notice that if $\chi^{\prime}$ is equivalent to $\chi$ then $\chi^{\prime}$ and $\chi$ both have the same order and conductor. We let $Y_{\mathbf{N} / \mathbf{L}}^{-}$denote any set of representatives of the set of equivalence classes of $X_{\mathrm{N} / \mathbf{L}}^{-}$modulo this relation. According to (4) and (17) and noticing that for $\chi \in X_{\mathbf{N} / \mathbf{L}}$ we have $\mathbf{L} \subseteq \mathbf{M}_{\chi} \subseteq \mathbf{N}$ (which implies $w_{\chi} \mid w_{\mathbf{N}}$ ), we have:

TheOrem 9. For any $\chi \in X_{\mathbf{N} / \mathbf{L}}^{-}$we have $w_{\mathbf{N}} L(0, \chi) \in \mathbf{Z}[\chi]$ and

$$
h_{\overline{\mathbf{N}}}^{-}=Q_{\mathbf{N}} w_{\mathbf{N}} \prod_{\chi \in Y_{\mathbf{N} / \mathbf{L}}^{-}} N_{\mathbf{Q}(\chi) / \mathbf{Q}}\left(\frac{1}{2^{m}} L(0, \chi)\right)
$$

Moreover, since

$$
N_{\mathbf{Q}(\chi) / \mathbf{Q}}\left(\frac{1}{2^{m}} L(0, \chi)\right)=\frac{1}{\left(2^{m} w_{\chi}\right)^{\phi_{\chi}}} \operatorname{Resultant}\left(\Phi_{\chi}(X), \sum_{k=0}^{n_{\chi}-1} c_{\chi}(k) X^{k}\right)
$$

where

$$
\Phi_{\chi}(X)=\prod_{\substack{k=1 \\ \operatorname{gcd}\left(k, n_{\chi}\right)=1}}^{n_{\chi}}\left(X-\zeta_{\chi}^{k}\right) \in \mathbf{Z}[X]
$$

denotes the minimal polynomial of $\zeta_{\chi}$ and where

$$
w_{\chi} L(0, \chi)=\sum_{k=1}^{\phi_{\chi}} b_{\chi}(k) \epsilon_{k}=\sum_{k=0}^{n_{\chi}-1} c_{\chi}(k) \zeta_{\chi}^{k} \in \mathbf{Z}[\chi]
$$

by computing with large rational integers we can easily compute the (usually very large) exact values of the rational numbers $N_{\mathbf{Q}(\chi) / \mathbf{Q}}\left(2^{-m} L(0, \chi)\right)$ as soon as we have computed the (small) coordinates $b_{\chi}(k) \in \mathbf{Z}$ of $w_{\chi} L(0, \chi)$

\section{$\S 5$. Examples of computation of relative class numbers of non- abelian normal CM-fields}

\subsection{Relative class numbers of some dihedral CM-fields}

Let $p \geq 3$ be an odd prime. Let $\mathbf{N}$ be a normal CM-field of degee $4 p$ whose Galois group is isomorphic to the dihedral group $D_{4 p}$ of ordre $4 p$. Hence, $\mathbf{N}=\mathbf{N}^{+} \mathbf{M}$ where $\mathbf{M}$ an imaginary biquadratic bicyclic field and $\mathbf{N}^{+}$ is a real dihedral field of degree $2 p$, cyclic of degree $p$ over the real quadratic subfield $\mathbf{L}$ of $\mathbf{M}$. There exists a positive rational integer $f \geq 1$ such that 
the conductor $\mathcal{F}_{\mathbf{N}^{+} / \mathbf{L}}$ of this extension $\mathbf{N}^{+} / \mathbf{L}$ is equal to the ideal $(f)$ of $\mathbf{L}$ (see [Mar] and [LPL]). We proved in [LOO] that $Q_{\mathbf{N}}=Q_{\mathbf{M}}, w_{\mathbf{N}}=w_{\mathbf{M}}$ and that $h_{\mathbf{M}}^{-}$divides $h_{\mathbf{N}}^{-}$. Hence, formula (18) applied to both $\mathbf{N}$ and $\mathbf{M}$ yields

$$
h_{\mathbf{N}}^{-} / h_{\mathbf{M}}^{-}=N_{\mathbf{Q}\left(\zeta_{p}\right) / \mathbf{Q}}\left(\frac{1}{4} L(0, \chi)\right) .
$$

Here, $\chi$ denotes any one of the $p-1$ primitive characters of order $2 p$ associated with the cyclic extension $\mathbf{N} / \mathbf{L}$ of degree $2 p$. Hence, $w_{\chi}=w_{\mathbf{N}}=w_{\mathbf{M}}$ divides 12 . We choose $\mathcal{B}=\left\{\zeta_{p}, \cdots, \zeta_{p}^{p-1}\right\}$ whose dual basis is (according to Lemma 3$) \mathcal{B}^{\perp}=\left\{\left(\zeta_{p}^{-1}-1\right) / p, \cdots,\left(\zeta_{p}^{-(p-1)}-1\right) / p\right\}$. We have

$$
\begin{aligned}
& w_{\mathbf{M}} L(0, \chi)=\sum_{k=1}^{p-1} b_{\chi}(k) \zeta_{p}^{k} \in \mathbf{Z}[\chi]=\mathbf{Z}\left[\zeta_{p}\right] \\
& \text { with } b_{\chi}(k)=\frac{w_{\mathbf{M}}}{p} \sum_{\substack{l=0 \\
l \neq(p-1) / 2}}^{p-1}\left(\zeta_{p}^{-k(2 l+1)}-1\right) L\left(0, \chi^{2 l+1}\right)
\end{aligned}
$$

(use $(7))$. Since the induced characters $\left(\chi^{2 l+1}\right)^{*}$ of the dihedral group $\operatorname{Gal}(\mathbf{N} / \mathbf{Q})$ of order $4 p$ are real valued, we have $b_{\chi}(p-k)=b_{\chi}(k)$ for $1 \leq k \leq(p-1) / 2$,

$$
L\left(0, \chi^{2(p-1-l)+1}\right)=L\left(0, \overline{\chi^{2 l+1}}\right)=\overline{L\left(0, \chi^{2 l+1}\right)}=L\left(0, \chi^{2 l+1}\right)
$$

and

$$
b_{\chi}(k)=\frac{2 w_{\mathbf{M}}}{p} \sum_{l=0}^{(p-3) / 2}(\cos (2 k(2 l+1) \pi / p)-1) L\left(0, \chi^{2 l+1}\right) .
$$

Thanks to the computation of good enough numerical approximations of the $L\left(0, \chi^{2 l+1}\right)$ for $0 \leq l \leq(p-3) / 2$ we can use $(21)$ to compute the exact values of the coordinates $b_{\chi}(k)$ of

$$
L(0, \chi)=\frac{2}{w_{\mathbf{M}}} \sum_{k=1}^{(p-1) / 2} b_{\chi}(k) \cos (2 k \pi / p) \in \mathbf{Q}\left(\zeta_{p}\right)^{+}=\mathbf{Q}(\cos (2 \pi / p))
$$

and $h_{\mathbf{N}}^{-} / h_{\mathbf{M}}^{-} \stackrel{\text { def }}{=}\left(h_{\mathbf{N} / \mathbf{M}}^{-}\right)^{2}$ is a perfect square with

$$
h_{\mathbf{N} / \mathbf{M}}^{-}=N_{\mathbf{Q}\left(\zeta_{p}\right)+/ \mathbf{Q}}\left(\frac{1}{4} L(0, \chi)\right) .
$$


Moreover, we have (see $[\mathrm{FQ}])$ :

$$
W_{\chi}=+1
$$

To make our construction of $\chi$ easy, we will choose examples such that

(i) $\mathbf{L}$ has class number one,

(ii) $\mathbf{M} / \mathbf{L}$ is unramified at all the finite places,

(iii) the conductor $\left(f_{+}\right)$of the cyclic extension $\mathbf{N}^{+} / \mathbf{L}$ of degree $p$ is of the form $(q)$ for some prime rational number $q$.

In that situation, $\chi$ is a primitive abelian Hecke character of order $2 p$ on the ray class group of conductor $\mathcal{F}=(q)$ of $\mathbf{L}$ and there exists a character $\chi_{+}$on $\left(\mathbf{A}_{\mathbf{L}} /(q)\right)^{*}$ of order $p$ (and trivial on the image of $\mathbf{Z}$ in this group) such that for any $\alpha \neq 0$ in the ring of algebraic integers $\mathbf{A}_{\mathbf{L}}$ of $\mathbf{L}$ we have $\chi((\alpha))=\nu(\alpha) \chi_{+}(\alpha)$, where $\nu(\alpha)$ is the sign of the norm of $\alpha$.

Example 1. Choose $p=5, \mathbf{L}=\mathbf{Q}(\sqrt{1633})=\mathbf{Q} \sqrt{23 \cdot 71})$ for which $y_{\mathbf{L}}=16332094890275578606272$ has 23 digits so that Shintani's method is useless, let $\mathbf{N}^{+}$be the only real dihedral field of degree $2 p=10$ for which $\mathcal{F}_{\mathbf{N}^{+} / \mathbf{L}}=(q)=(59)$ and take $\mathbf{M}=\mathbf{Q}(\sqrt{-23}, \sqrt{-71})$, for which $h_{\mathbf{M}}^{-}=21$ and $w_{\mathbf{M}}=2$. Hence, $\mathbf{N}=\mathbf{N}^{+} \mathbf{M}$ is a dihedral CM-field of degree $4 p=20$. Since $q$ is inert in $\mathbf{L}$, then (see [Lou6] and [LPL]):

$$
\chi((\alpha))=\nu(\alpha) \phi(\alpha)
$$

for some character $\phi$ of order $p=5$ on the cyclic group $\left(\mathbf{A}_{\mathbf{L}} /(q)\right)^{*}$ of order $q^{2}-1$. To make it explicit which $\chi$ we used we chose $\phi$ the one for which $\phi\left(\left(101+\sqrt{d_{\mathbf{L}}}\right) / 2\right)=\zeta_{p}=\exp (2 \pi i / 5)$. According to our numerical computation we have $b_{\chi}(1)=-320, a_{\chi}(2)=-240$,

$$
L(0, \chi)=140-20 \sqrt{5}
$$

and $h_{\mathbf{N} / \mathbf{M}}^{-}=N_{\mathbf{Q}(\sqrt{5}) / \mathbf{Q}}(35-5 \sqrt{5})=1100$.

Example 2. Choose $p=5, \mathbf{L}=\mathbf{Q}(\sqrt{21})$, let $\mathbf{N}^{+}$be the only real dihedral field of degree $2 p=10$ for which $\mathcal{F}_{\mathbf{N}^{+} / \mathbf{L}}=(q)=\left(10^{6}+151\right)$ and take $\mathbf{M}=\mathbf{Q}(\sqrt{-3}, \sqrt{-7})$, for which $h_{\mathbf{M}}^{-}=1$ and $w_{\mathbf{M}}=6$. Hence, $\mathbf{N}=\mathbf{N}^{+} \mathbf{M}$ is a dihedral CM-field of degree $4 p=20$. Since $(q)=\mathcal{Q} \mathcal{Q}^{\prime}$ splits in $\mathbf{L}$ then (see [Lou6] and [LPL]):

$$
\chi((\alpha))=\nu(\alpha) \phi\left(\alpha / \alpha^{\prime}\right)
$$


for some character $\phi$ of order $p=5$ on the cyclic group $\left(\mathbf{A}_{\mathbf{L}} / \mathcal{Q}\right)^{*}$ of order $q-1$, group which is canonically isomorphic to $(\mathbf{Z} / q \mathbf{Z})^{*}$ (here $\alpha^{\prime}$ is the conjugate of $\alpha$ in $\mathbf{L}$ ). To make it explicit which $\chi$ we used we chose $\mathcal{Q}=q \mathbf{Z}+\left(\left(716593+\sqrt{d_{\mathbf{L}}}\right) / 2\right) \mathbf{Z}$ and $\phi$ is the one for which $\phi(11)=$ $\zeta_{p}=\exp (2 \pi i / 5)$. According to our numerical computation we have $b_{\chi}(1)=$ $-4392096, b_{\chi}(2)=-3650880$,

$$
L(0, \chi)=670248-61768 \sqrt{5}
$$

$($ for $\cos (2 \pi / 5)=(\sqrt{5}-1) / 4$ and $\cos (4 \pi / 5)=-(\sqrt{5}+1) / 4)$, and $h_{\mathbf{N} / \mathbf{M}}^{-}=$ $N_{\mathbf{Q}(\sqrt{5}) / \mathbf{Q}}(167562-15442 \sqrt{5})=26884747024$.

Example 3. Finally, choose $p=41, \mathbf{L}=\mathbf{Q}(\sqrt{69})$, let $\mathbf{N}^{+}$be the only real dihedral field of degree $2 p=82$ for which $\mathcal{F}_{\mathbf{N}^{+} / \mathbf{L}}=(q)=(2297)$ and take $\mathbf{M}=\mathbf{Q}(\sqrt{-3}, \sqrt{-23})$, for which $h_{\mathbf{M}}^{-}=1$. Hence, $\mathbf{N}=\mathbf{N}^{+} \mathbf{M}$ is a dihedral CM-field of degree $4 p=164$. The construction of $\chi$ is similar to that of example 2. To make it explicit which $\chi$ we used we chose $\mathcal{Q}=$ $q \mathbf{Z}+\left(\left(2527+\sqrt{d_{\mathbf{L}}}\right) / 2\right) \mathbf{Z}$ and $\phi$ is the one for which $\phi(5)=\zeta_{p}=\exp (2 \pi i / 41)$. According to our numerical computation we have

$$
L(0, \chi)=\sum_{k=1}^{40} b_{k} \zeta_{41}^{k}
$$

with $b_{41-k}=b_{k}$ and the following Table:

\begin{tabular}{|l|rrrrr|}
\hline$k$ & 1 & 2 & 3 & 4 & 5 \\
\hline$b_{k}$ & -4008 & -4000 & -4028 & -4076 & -4260 \\
\hline$k$ & 6 & 7 & 8 & 9 & 10 \\
\hline$b_{k}$ & -4092 & -4100 & -3964 & -3664 & -3868 \\
\hline$k$ & 11 & 12 & 13 & 14 & 15 \\
\hline$b_{k}$ & -3820 & -3964 & -4024 & -4044 & -4700 \\
\hline$k$ & 16 & 17 & 18 & 19 & 20 \\
\hline$b_{k}$ & -4384 & -4012 & -4068 & -3960 & -3896 \\
\hline
\end{tabular}

Hence, $h_{\mathbf{N}}^{-} / h_{\mathbf{M}}^{-}=\left(h_{\mathbf{N} / \mathbf{M}}^{-}\right)^{2}$ with $h_{\mathbf{N} / \mathbf{M}}^{-}=4780651139182896937025122$ $72645030255859142700365392814996559 \approx 4 \cdot 10^{59}$. This huge relative class number could not have been computed with the method developed in [Lou6, Theorem 7]. 
Remarks 10. Our present method for computing relative class numbers is much more efficient than the method developed in [Lou6, Theorem 7]. There, we had to compute very good approximations of all the $S_{M}\left(\chi^{l}\right)$ (defined in Theorem 5) prior to taking their product to deduce the value of a relative class number. Here, we only have to compute fair approximations of these $S_{M}\left(\chi^{l}\right)$ prior to taking linear combinations of them to deduce the exact values of the coordinates of $L(0, \chi)$. Then, we compute the value of the relative class number by computing the norm of the algebraic number $L(0, \chi)$.

Popular tools like Pari or Kash, which compute class groups of numbers fields $\mathbf{N}$, are useless for computing unconditionally (relative) class numbers of CM-fields $\mathbf{N}$ of degrees $\geq 12$ (or so). Moreover, these softwares usually require the user to determine a polynomial $P_{\mathbf{N}}(X)$ such that $\mathbf{N}$ is generated by a root of $P_{\mathbf{N}}(X)$, but such polynomials $P_{\mathbf{N}}(X)$ are far from being easy to compute, even for dihedral fields.

However, the latest versions of Pari can now compute approximations of $L$-functions (in much the same way as in Subsection 3.1) and Artin roots numbers (by going back to their definition) and can be conveniently combined with the techniques developed in this paper. In fact, for the forthcoming situation we used Pari to compute the coefficients $a_{n}(\chi)$ of the Dirichlet series $L(s, \chi)$ whose exact values at $s=0$ we wanted to compute.

\subsection{Relative class numbers of some CM-fields of degree 24}

Let $\mathbf{N}$ be a normal CM-field of degree 24 with Galois group isomorphic to $S L_{2}\left(F_{3}\right)$, the special linear group over the finite field $F_{3}$ with three elements (see [Lou3, Section 5] and [LLO]). Let $\mathbf{N}^{+}$denote the maximal totally real subfield of $\mathbf{N}$. Then $\mathbf{N}^{+} / \mathbf{Q}$ is a normal extension of degree 12 with Galois group isomorphic to the alternating group $\mathcal{A}_{4}$ of degree 4 and order 12 , and $\mathbf{N}$ is a quaternion octic extension of some cyclic cubic field $\mathbf{F}$. Let $f_{\mathbf{F}}$ denote the conductor of $\mathbf{F}$. We let $\mathbf{L} / \mathbf{F}$ denote a fixed quadratic subextension of the three quadratic subextensions $\mathbf{L}_{i} / \mathbf{F}$ of the bicyclic biquadratic extension $\mathbf{N}^{+} / \mathbf{F}$. (Notice that $\mathbf{L} / \mathbf{Q}$ is not normal and that the three $\mathbf{L}_{i}$ are conjugate). Then, $\mathbf{N} / \mathbf{L}$ is a cyclic quartic extension and we let $\chi$ denote any one of the two conjugate characters of order four associated with this cyclic quartic extension $\mathbf{N} / \mathbf{L}$. An (incomplete) lattice of subfields is given in the following Diagram: 


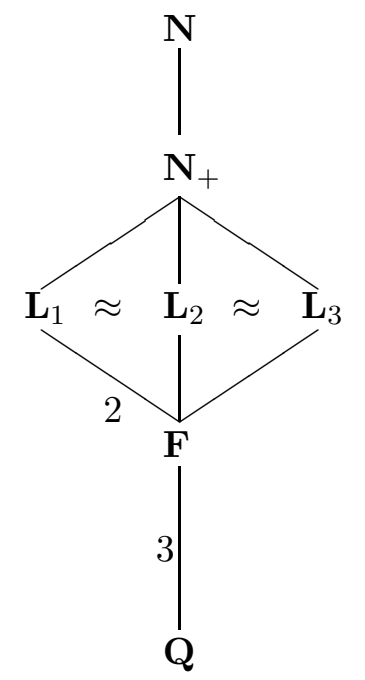

$$
\begin{aligned}
& \operatorname{Gal}(\mathbf{N} / \mathbf{Q})=S L_{2}\left(F_{3}\right) \\
& \operatorname{Gal}(\mathbf{N} / \mathbf{F})=\mathcal{Q}_{8} \\
& \operatorname{Gal}\left(\mathbf{N}^{+} / \mathbf{Q}\right)=\mathcal{A}_{4} \\
& \operatorname{Gal}(\mathbf{F} / \mathbf{Q})=\mathcal{C}_{3} \\
& \operatorname{Gal}\left(\mathbf{N} / \mathbf{L}_{i}\right)=\mathcal{C}_{4}
\end{aligned}
$$

and we have

$$
h_{\mathbf{N}}^{-}=Q_{\mathbf{N}} w_{\mathbf{N}} N_{\mathbf{Q}(i) / \mathbf{Q}}\left(2^{-6} L(0, \chi)\right) .
$$

Now, since $\mathbf{F}$ is the maximal abelian subfield of $\mathbf{N}$ then $w_{\mathbf{N}}=2$ and $2 L(0, \chi) \in \mathbf{Z}[i]$. Since the character of $\operatorname{Gal}(\mathbf{N} / \mathbf{F})$ induced by $\chi$ is the irreducible character of degree two of the quaternion octic group which is real valued, then $L(0, \chi)$ is real, hence rational and we get $L(0, \chi) \in \frac{1}{2} \mathbf{Z}$ and

$$
h_{\mathbf{N}}^{-}=\left(Q_{\mathbf{N}} / 2\right)(L(0, \chi) / 32)^{2},
$$

which implies $L(0, \chi) \in 32 \mathbf{Z}$, and $L(0, \chi)=-A_{\chi} L(1, \chi) / \pi^{3}<0$. Moreover, if $h_{\mathbf{N}}^{-}$is odd then $Q_{\mathbf{N}}=2, h_{\mathbf{N}}^{-}=(L(0, \chi) / 32)^{2}$ is a perfect square and $L(0, \chi) \notin 64 \mathbf{Z}$. Conversely, if $L(0, \chi) \in 32 \mathbf{Z} \backslash 64 \mathbf{Z}$ then $Q_{\mathbf{N}}=2, h_{\mathbf{N}}^{-}$is odd and $h_{\mathbf{N}}^{-}=(L(0, \chi) / 32)^{2}$ is a perfect square. Now, for any number field $\mathbf{E}$, let $C l_{\mathbf{E}}$ and $C l_{\mathbf{E}}^{+}$denote the 2-Sylow subgroups of the ideal class group and narrow ideal class groups of $\mathbf{E}$, respectively. According to [LLO], if $h_{\mathbf{N}}^{-}$is odd then $C l_{\mathbf{F}}$ and $C l_{\mathbf{F}}^{+}$are both isomorphic to $(\mathbf{Z} / 2 \mathbf{Z})^{2}, C l_{\mathbf{L}}$ is isomorphic to $\mathbf{Z} / 2 \mathbf{Z}, C l_{\mathbf{L}}^{+}$is isomorphic to $\mathbf{Z} / 4 \mathbf{Z}$ and $\mathbf{N}^{+}$is the Hilbert 2-class field of $\mathbf{F}$ and $\mathbf{N}$ is the Hilbert 2-class field of $\mathbf{L}$. In particular, $A_{\chi}=\sqrt{d_{\mathbf{L}} / \pi^{6}}=\sqrt{d_{\mathbf{F}}^{2} / \pi^{6}}=\sqrt{f_{\mathbf{F}}^{4} / \pi^{6}}=f_{\mathbf{F}}^{2} / \pi^{3}$ and

$$
W_{\chi}=-1
$$

(for the abelian extension $\mathbf{N} / \mathbf{L}$ is unramified at all the finite places of $\mathbf{L}$, but ramified at the six infinite real places of $\mathbf{L}$ ). 
Now, in using class field theory, the reader would prove the following result which, together with the results of Section 3.1, enables us to compute the exact values of $L(0, \chi)$ for such characters $\chi$ :

Proposition 11. Let $\mathbf{F}, \mathbf{L}, \mathbf{N}$ and $\chi$ be as above. Assume that $f_{\mathbf{F}}=$ $q \equiv 1 \quad(\bmod 6)$ is prime, that the narrow and ordinary class groups of $\mathbf{F}$ are isomorphic to $(\mathbf{Z} / 2 \mathbf{Z})^{2}$, and that the narrow and ordinary class groups of $\mathbf{L}$ are isomorphic to $\mathbf{Z} / 4 \mathbf{Z}$ and $\mathbf{Z} / 2 \mathbf{Z}$, respectively (see [Lou3, Pro. 16]).

1. if $(p)=\mathbf{P}$ is inert in $\mathbf{F}$ then $(p)=\mathbf{P} \mathbf{A}_{\mathbf{L}}=\mathcal{P} \mathcal{P}^{\prime}$ splits in $\mathbf{L} / \mathbf{F}$, and setting $\epsilon_{p}=\chi(\mathcal{P})=\chi\left(\mathcal{P}^{\prime}\right)= \pm 1$, we have

$$
a_{p^{k}}(\chi)= \begin{cases}0 & \text { if } 3 \text { does not divide } k \\ \epsilon_{p}^{k / 3}(k+3) / 3 & \text { if } 3 \text { divides } k\end{cases}
$$

and $\epsilon_{p}=+1$ if and only if $\mathcal{P}$ is principal in the narrow sense (notice that $\mathcal{P}$ is always principal in the ordinary sense).

2. if $p=q$ is totally ramified in $\mathbf{F}$, say $p \mathbf{A}_{\mathbf{F}}=\mathbf{P}^{3}$, then $\mathbf{P} \mathbf{A}_{\mathbf{L}}=\mathcal{P} \mathcal{P}^{\prime}$ splits in $\mathbf{L}$, and setting $\epsilon_{p}=\chi(\mathcal{P})=\chi\left(\mathcal{P}^{\prime}\right)= \pm 1$, we have

$$
a_{p^{k}}(\chi)=\epsilon_{p}^{k}(k+1)
$$

and $\epsilon_{p}=+1$ if and only if $\mathcal{P}$ is principal in the narrow sense (notice that $\mathcal{P}$ is always principal in the ordinary sense).

3. Assume that $(p)=\mathbf{P}_{1} \mathbf{P}_{2} \mathbf{P}_{3}$ splits in $\mathbf{F}$. Then either these three ideals are principal in $\mathbf{F}$ or none of them is principal in $\mathbf{F}$.

(a) If the three $\mathbf{P}_{i}$ 's are principal in $\mathbf{F}$ then each $\mathbf{P}_{i} \mathbf{A}_{\mathbf{L}}=\mathcal{P}_{i} \mathcal{P}_{i}^{\prime}$ splits in $\mathbf{L} / \mathbf{F}$, and setting $\epsilon_{p}=\chi\left(\mathcal{P}_{i}\right)=\chi\left(\mathcal{P}_{i}^{\prime}\right)= \pm 1$ which does not depends on $i$, we have

$$
a_{p^{k}}(\chi)=\epsilon_{p}^{k}(k+5)(k+4)(k+3)(k+2)(k+1) / 120
$$

and $\epsilon_{p}=+1$ if and only if $\mathcal{P}_{i}$ is principal in the narrow sense (notice that $\mathcal{P}_{i}$ is always principal in the ordinary sense).

(b) If none of the $\mathbf{P}_{i}$ is principal in $\mathbf{F}$ then two of these prime ideals $\mathbf{P}_{1} \mathbf{A}_{\mathbf{L}}=\mathcal{P}_{1}$ and $\mathbf{P}_{2} \mathbf{A}_{\mathbf{L}}=\mathcal{P}_{2}$ are inert in $\mathbf{L} / \mathbf{F}$ and the third one $\mathbf{P}_{3} \mathbf{A}_{\mathbf{L}}=\mathcal{P}_{3} \mathcal{P}_{3}^{\prime}$ splits in $\mathbf{L} / \mathbf{F}$. We have $\chi\left(\mathcal{P}_{1}\right)=\chi\left(\mathcal{P}_{2}\right)=-1$, $\chi\left(\mathcal{P}_{3}^{\prime}\right)=\overline{\chi\left(\mathcal{P}_{3}\right)}= \pm i$ and

$$
a_{p^{k}}(\chi)= \begin{cases}0 & \text { if } k \text { is odd } \\ (-1)^{k / 2}((k / 2)+1)((k / 2)+2) / 2 & \text { if } k \text { is even } .\end{cases}
$$


For example, using Pari to decide whether a given ideal $\mathcal{P}$ of the sextic field $\mathbf{L}$ is principal in the ordinary or narrow senses, we computed the following Table of relative class numbers:

\begin{tabular}{|l|l|l|l|}
\hline$f_{\mathbf{F}}$ & $P_{\mathbf{F}}(X), P_{\mathbf{L}}(X)$ & $L(0, \chi)$ & $h_{\mathbf{N}}^{-}$ \\
\hline 163 & $P_{\mathbf{F}}(X)=x^{3}-x^{2}-54 x+169$ & & \\
& $P_{\mathbf{L}}(X)=x^{6}-3 x^{5}-11 x^{4}+27 x^{3}-3 x^{2}-11 x+1$ & -32 & 1 \\
349 & $P_{\mathbf{F}}(X)=x^{3}-x^{2}-116 x+517$ & -96 & $3^{2}$ \\
& $P_{\mathbf{L}}(X)=x^{6}-3 x^{5}-17 x^{4}+39 x^{3}-3 x^{2}-17 x+1$ & & \\
397 & $P_{\mathbf{F}}(X)=x^{3}-x^{2}-132 x+544$ & -96 & $3^{2}$ \\
& $P_{\mathbf{L}}(X)=x^{6}-26 x^{4}+93 x^{2}-4$ & & \\
853 & $P_{\mathbf{F}}(X)=x^{3}-x^{2}-284 x-1011$ & -352 & $11^{2}$ \\
& $P_{\mathbf{L}}(X)=x^{6}-3 x^{5}-53 x^{4}+111 x^{3}+705 x^{2}-761 x-91$ & & \\
937 & $P_{\mathbf{F}}(X)=x^{3}-x^{2}-312 x+2221$ & -608 & $19^{2}$ \\
\hline
\end{tabular}

Notice that in these five cases it holds $L(0, \chi) \in 32 \mathbf{Z} \backslash 64 \mathbf{Z}$. Hence, according to $(23)$, we have $Q_{\mathbf{N}}=2$ and $h_{\mathbf{N}}^{-}=(L(0, \chi) / 32)^{2}$ is a perfect odd square. Such computations for the 23 CM-fields $\mathbf{N}$ associated with the 23 cyclic cubic fields $\mathbf{F}$ whose conductors $f_{\mathbf{F}}$ are listed in [Lou3, Prop. 16] enable us to prove:

THEOREM 12. There exists only one normal CM-field of degree 24 with Galois group isomorphic to $S_{2}\left(F_{3}\right)$ with class number one: the CMfield $\mathbf{N}$ associated with the cyclic cubic field $\mathbf{F}$ of conductor $f_{\mathbf{F}}=163$.

Proof. According to our relative class number computations the cyclic cubic field $\mathbf{F}$ of conductor $f_{\mathbf{F}}=163$ is the only field (out of the 23 cyclic cubic fields $\mathbf{F}$ whose conductors $f_{\mathbf{F}}$ are listed in [Lou3, Prop. 16]) for which $h_{\mathbf{N}}^{-}=1$. It remains to prove that $h_{\mathbf{N}^{+}}=1$. To this end, we use Odlyzko's bounds on discriminants . First, since $h_{\mathbf{N}}^{-}=1$ is odd and since $Q_{\mathbf{N}}=2$, then $h_{\mathbf{N}^{+}}$is odd (see [Wa, Exercise 10.7 and proof of Th. 10.2]). Second, since $\mathbf{N}^{+} / \mathbf{F}$ is bicyclic biquadratic and since the three $\mathbf{L}_{i}$ are conjugate, if some odd prime $p$ divides $h_{\mathbf{N}^{+}}$then $p^{3}$ divides $h_{\mathbf{N}^{+}}$(see [Lem]). Therefore, if we had $h_{\mathbf{N}^{+}}>1$ then some odd prime $p$ would divide $h_{\mathbf{N}^{+}}$and we could construct a totally real number field $\mathbf{E}$ of degree $n_{\mathbf{E}}=12 p^{3} \geq 12 \cdot 3^{3}=324$ and root discriminant $d_{\mathbf{E}}^{1 / n_{\mathbf{E}}}=d_{\mathbf{F}}^{1 / n_{\mathbf{F}}}=163^{2 / 3}<30$, which contradicts Odlyzko's lower bound $d_{\mathbf{E}}^{1 / n_{\mathbf{E}}}>30$ for root discriminants of totally real number fields of degree $\geq 100$ (see [Wa, Chapter 11]). Hence $h_{\mathbf{N}^{+}}=1$. 


\subsection{Relative class numbers of some CM-fields of degree 42}

We refer the reader to $[\mathrm{LPCK}]$ for examples of computation of Artin root numbers $W_{\chi}$ and values at $s=0$ of $L$-functions associated with characters of order 14 on ray class groups of real cyclic cubic fields $\mathbf{L}$ and for the proof of the following result similar to Theorem 12:

THEOREM 13. There is no non-abelian normal CM-field of degree 42 with relative class number one.

\section{REFERENCES}

[Cas] P. Cassou-Noguès, Valeurs aux entiers négatifs des fonctions zêta et fonctions zêta p-adiques, Invent. Math., 51 (1979), 29-59.

[CK] K.-Y. Chang and S.-H. Kwon, The non-abelian normal CM-fields of degree 36 with calss number one, Acta Arith., to appear.

[CS1] J. Coates and W. Sinnott, On p-adic L-functions over real quadratic fields, Invent. Math., 25 (1974), 253-279.

[CS2] Integrality properties of the values of partial zeta functions, Proc. London Math. Soc., 34 (1977), 365-384.

[FQ] A. Fröhlich and J. Queyrut, On the functional equation of the Artin L-function for characters of real representations, Invent. Math., 20 (1973), 125-138.

[Fro] A. Fröhlich, Artin-root numbers and normal integral bases for quaternion fields, Invent. Math., 17 (1972), 143-166.

[Hid] H. Hida, Elementary theory of $L$-functions and Eisenstein series, London Mathematical Society, Student Texts 26,, Cambridge University Press, 1993.

[Lan] S. Lang, Algebraic Number Theory, Springer-Verlag, Grad. Texts Math. 110, Second Edition, 1994.

[Lef] Y. Lefeuvre, Corps diédraux à multiplication complexe principaux, Ann. Inst. Fourier (Grenoble), 50 (2000), 67-103.

[Lem] F. Lemmermeyer, Kuroda's class number formula, Acta Arith., 66 (1994), $245-260$.

[LLO] F. Lemmermeyer, S. Louboutin and R. Okazaki, The class number one problem for some non-abelian normal CM-fields of degree 24, J. Théor. Nombres Bordeaux, 11 (1999), 387-406.

[LO] S. Louboutin and R. Okazaki., The class number one problem for some non-abelian normal CM-fields of 2-power degrees, Proc. London. Math. Soc. (3), 76 (1998), 523-548.

[LOO] S. Louboutin, R. Okazaki and M. Olivier, The class number one problem for some non-abelian normal CM-fields, Trans. Amer. Math. Soc., 349 (1997), 3657-3678.

[Lou1] S. Louboutin, Calcul du nombre de classes des corps de nombres, Pacific J. Math., 171 (1995), 455-467.

[Lou2] Computation of relative class numbers of CM-fields, Math. Comp., 66 (1997), 173-184. 
[Lou3] Upper bounds on $|L(1, \chi)|$ and applications, Canad. Math. J. (4), 50 (1998), 794-815.

[Lou4] Computation of relative class numbers of imaginary abelian number fields, Experimental Math., 7 (1998), 293-303.

[Lou5] Sur le calcul numérique des constantes des équation fonctionnelles des fonctions L associées aux caractères impairs, C. R. Acad. Sci. Paris, 329 (1999), $347-350$.

[Lou6] Computation of relative class numbers of CM-fields by using Hecke L-functions, Math. Comp., 69 (2000), 371-393.

[Lou7] - Explicit bounds for residues of Dedekind zeta functions, values at $s=1$ of L-functions and relative class numbers, J. Number Theory, 85 (2000), $263-282$.

[Lou8] - Formulae for some Artin root numbers, Tatra Mountains Math. Publ, to appear.

[LP] S. Louboutin and Y.-H. Park, Class number problems for dicyclic CM-fields, Publ. Math. Debrecen, 57 (2000), 283-295.

[Park] Y.-H. Park, The calss number one problem for the non-abelian normal CM-fields of degree 24 and 40, Acta Arith., to appear.

[LPCK] S. Louboutin, Y.-H. Par, K.-Y. Chang and S.-H. Kwon, The class number one problem for the non abelian normal CM-fields of degree 2pq, Preprint (1999).

[LPL] S. Louboutin, Y.-H. Park and Y. Lefeuvre, Construction of the real dihedral number fields of degree 2p. Applications, Acta Arith., 89 (1999), 201-215.

[Mar] J. Martinet, Sur l'arithmétique des extensions à groupe de Galois diédral d'ordre $2 p$, Ann. Inst. Fourier (Grenoble), 19 (1969), 1-80.

[Odl] A. M. Odlyzko, Some analytic estimates of class numbers and discriminants, Invent. Math., 29 (1975), 275-286.

[Oka1] R. Okazaki, On evaluation of L-functions over real quadratic fields, J. Math. Kyoto Univ., 31 (1991), 1125-1153.

[Oka2] - An elementary proof for a theorem of Thomas and Vasquez, J. Number Theory, 55 (1995), 197-208.

[Pes] M. Pestour, Valeurs en $s=1$ de fonctions L, Acta Arith., 78 (1997), 367-376.

[Shi] T. Shintani, On evaluation of zeta functions of totally real algebraic number fields at non-positive integers, J. Fac. Sci. Univ. Tokyo, 23 (1976), 373-471.

[Tsu] S. Tsuyumine, On values of L-functions of totally real algebraic number fields at integers,, Acta Arith., 76 (1996), 359-392.

[Wa] L.C. Washington, Introduction to Cyclotomic Fields, Springer-Verlag, Grad. Texts Math. 83, Second Edition, 1997.

[Zag1] D. Zagier, A Kronecker limit formula for real quadratic fields, Math. Ann., 213 (1975), 153-184.

[Zag2] - Nombres de classes et fractions continues, Soc. Math. de France, Astérisque., 24-25 (1975), 81-97.

[Zag3] - Valeurs des fonctions zêta des corps quadratiques réels aux entiers négatifs,, Soc. Math. de France, Astérisque., 41-42 (1977), 135-151. 
Institut de Mathématiques de Luminy UPR 9016

163, avenue de Luminy

Case 907

13288 Marseille Cedex 9, France

loubouti@iml.univ-mrs.fr 